\title{
Aharonov-Bohm cages in two-dimensional structures
}

\author{
Julien Vidal ${ }^{1}$, Rémy Mosseri ${ }^{1}$ and Benoit Douçot ${ }^{2}$ \\ ${ }^{1}$ Groupe de Physique des Solides, \\ ${ }^{2}$ Laboratoire de Physique Théorique des Hautes Energies, \\ Universités P. et M. Curie P6 et D. Diderot P7, Tour 23, 2 place Jussieu, 75251 Paris Cedex 05
}

\begin{abstract}
We present an extreme localization mechanism induced by a magnetic field for tight-binding electrons in two-dimensional structures. This spectacular phenomenon is investigated for a large class of tilings (periodic, quasiperiodic, or random). We are led to introduce the Aharonov-Bohm cages defined as the set of sites eventually visited by a wavepacket that can, for particular values of the magnetic flux, be bounded. We finally discuss the quantum dynamics which exhibits an original pulsating behaviour.
\end{abstract}

PACS numbers: 7100, 7210, 7335 .

Over the past two decades, the behaviour of electrons in two-dimensional structures under a magnetic field has been of special interest in condensed matter physics. In situations where electron-electron interactions are dominant (low carrier density heterostructures for instance), a strong magnetic field has been shown to induce new collective states such as fractional quantum Hall states [1] or Wigner crystals [2]. But even when electrons may be considered as weakly interacting, fascinating phenomena may take place in the presence of a spatially modulated potential besides the external magnetic field. In the case of a random potential, one obtains the integer quantum Hall effect [3] which is characterized by a complex oneparticle spectrum of localized states and a discrete set of eigenenergies associated to extended states [4]. If the modulated potential corresponds to a periodic lattice, a subtle competition between this periodicity and the area scale imposed by the magnetic field induces remarkable features for the one-particle spectrum [5], illustrated by the well-known Hofstadter's butterfly-like pattern [6]. The physics of this magnetically induced frustration has also generated many experiments on submicronic structures such as superconducting networks [7]. They have in turn triggered investigations of several different twodimensional structures, like the triangular lattice $[8]$, the honeycomb tiling [9], the quasiperiodic tilings [10], and even fractal networks [11] 12.

In this letter, we discuss a new effect of extreme localization in a large class of rhombus tilings. For particular values of the magnetic field, the set of sites visited by an initially localized wavepacket can be bounded due to Aharonov-Bohm (A.B.) destructive interferences [13]. We are thus led to introduce the notion of $\mathcal{A} \cdot \mathcal{B}$. cages. Our aim is to analyze the relationship between this phenomenon, the tiling geometry, the quantum dynamics, and the spectrum. We therefore pay attention to a periodic tiling with hexagonal symmetry [14, here denoted $\mathcal{T}_{3}$ (see figure 1). We show that its spectrum is related to that of the triangular lattice through a simple analytical transformation, which allows to compute the eigenspectrum for rational values of the magnetic flux. When the magnetic flux per rhombic plaquette equals half the flux quantum, one faces a rather exotic effect for an infinite periodic tiling, since the density of states degenerates into three $\delta$-peaks and the electron motion becomes bounded. We also present another example of an infinite periodic tiling denoted $\mathcal{T}_{4}$, which, while having a more complex geometry, displays the same properties as $\mathcal{T}_{3}$.

Other structures, like quasiperiodic or random tilings, can contain some bounded cages due to these particular star configurations, but generically, one does not expect a highly degenerated discrete spectrum as for $\mathcal{T}_{3}$ and $\mathcal{T}_{4}$. In both cases, cages induce interesting instances of wavepacket time evolution with well characterized pulsing states.

Let us first examine $\mathcal{T}_{3}$, a bipartite periodic hexagonal structure with 3 sites per unit cell (figure 1), one 6 -fold coordinated (denoted $A$ ) and two 3-fold coordinated (denoted $B$ and $C$ ). We consider an tight-binding hamiltonian defined by:

$$
\mathcal{H}=\sum_{<i, j>} t_{i j}|i><j|
$$

where $t_{i j}=1$ if $i$ and $j$ are nearest neighbours, 0 otherwise, and $\mid i>$ is a localized orbital on site $i$. In the presence of a magnetic field $\mathbf{H}$, the hopping terms are modified by phase factors involving the vector potential A 15. Let $\gamma_{i j}$ be the phase factor between sites $i$ and $j$ :

$$
\gamma_{i j}=\frac{2 \pi}{\phi_{0}} \int_{i}^{j} \mathbf{A} \cdot d \mathbf{l}
$$

where $\phi_{0}=h c / e$ is the flux quantum. We first consider a uniform magnetic field $\mathbf{H}=\mathrm{Hz}$ in the Landau gauge: $\mathbf{A}=H(0, x, 0)$. As shown below, the whole spectrum only depends on the reduced flux $f=\phi / \phi_{0}$ where $\phi=H a^{2} \sqrt{3} / 2$ is the magnetic flux through an elementary rhombus. Taking into account the translational in- 
variance in the $y$ direction due to the gauge choice, the eigenfunctions write:

$$
\varphi(x, y)=\psi(x) e^{i k_{y} y}, k_{y} \in[0,2 \pi / a \sqrt{3}]
$$

which allows to map the spectrum onto the solution of an effective one-dimensional problem:

$$
\begin{aligned}
\left(\varepsilon^{2}-6\right) \psi_{m}= & 2 \cos \left(\frac{\gamma}{2}\right)\left[2 \cos \left(\frac{3 \gamma}{2}\left(m+\frac{1}{2}\right)+\kappa\right) \psi_{m+1}\right. \\
& +2 \cos \left(\frac{3 \gamma}{2}\left(m-\frac{1}{2}\right)+\kappa\right) \psi_{m-1} \\
& \left.+2 \cos (3 \gamma m+2 \kappa) \psi_{m}\right]
\end{aligned}
$$

where $\psi_{m}=\psi_{A}(x)$ for $x=3 m a / 2(m \in \mathbf{Z})$, is the wave function at the $A$-type sites, $\kappa=k_{y} \sqrt{3} / 2, \gamma=2 \pi f$, and $\varepsilon$ is the energy for the two-dimensional original problem. Note that this mapping is only valid for $\varepsilon \neq 0$ (the $\varepsilon=0$ case is briefly adressed below). For the triangular lattice, a similar equation has been derived by Claro and Wannier [8]:

$$
\begin{aligned}
\varepsilon_{T} \psi_{m}= & 2 \cos \left(\gamma_{T}\left(m+\frac{1}{2}\right)+\kappa\right) \psi_{m+1} \\
& +2 \cos \left(\gamma_{T}\left(m-\frac{1}{2}\right)+\kappa\right) \psi_{m-1} \\
& +2 \cos \left(2 \gamma_{T} m+2 \kappa\right) \psi_{m}
\end{aligned}
$$

with similar notations. So, the $\mathcal{T}_{3}$ spectrum can be obtained from the triangular lattice one by choosing $\gamma_{T}=3 \gamma / 2$; the eigenenergies of the two tilings being related by:

$$
\varepsilon^{2}-6=2 \cos \left(\frac{\gamma}{2}\right) \varepsilon_{T}
$$

This extends a classical result on bipartite structures to the case of a uniform magnetic field. Since the tiling is made up of rhombi, the spectrum is symmetric $(\varepsilon \leftrightarrow-\varepsilon)$. Moreover, eq. (体) displays a translation symmetry $f \rightarrow f+n(n \in \mathbf{N})$ and a reflection invariance about half-integer values of $f$. Thanks to these symmetries, we can limit our analysis to $0 \leq f \leq 1 / 2$. For rational values of $f=p / q$ ( $p, q$ mutually prime), the system (何) becomes closed after a translation by $q$ periods and the spectrum is made up of $2 q$ bands. Note that, when $q=3 q^{\prime}\left(q^{\prime} \in \mathbf{N}\right)$, this period is reduced by a factor 3 (and the spectrum has only $2 q^{\prime}$ bands). An interesting example occurs for $f=1 / 3$ where the spectrum displays two symmetric bands extending from \pm 3 to 0 , and is therefore gapless. In addition, for any value of the magnetic field, a close inspection of the secular equations system for each type of site shows that the spectrum contains a highly degenerated eigenenergy (flat band) at $\varepsilon=0$ with weight $1 / 3$. Figure 2 shows the $\mathcal{T}_{3}$ spectrum support versus $f$. The most remarkable point in this context, is that for $f=1 / 2$, the spectrum collapses into three eigenvalues $\varepsilon=0$ and $\varepsilon^{ \pm}= \pm \sqrt{6}$ (see eq. (6)).

One way to analyze the link between the spectrum and the quantum dynamics is to study the spectral properties at a local level. The magnetic field being uniform, the whole spectrum is recovered from the three local density of states (LDOS); furthermore, by symmetry, the two 3fold coordinated sites $(B$ and $C$ ) are equivalent, so that we just need to study two different types of local environment. It is then suitable to shift from the above Landau gauge to an equivalent cylindrical symmetric gauge given by $\mathbf{A}=(H / 2)(-y, x, 0)$.

We proceed to an analytic Lanczos tridiagonalization of local clusters, along the recursion method algorithm [16]. An effective semi-infinite chain is generated, with normalized orbitals $|n\rangle$ and hopping terms $\left(a_{n}, b_{n}\right)$, according to the three-term recurrence relation:

$$
b_{n+1}|n+1\rangle=\left(\mathcal{H}-a_{n}\right)|n\rangle-b_{n}|n-1\rangle
$$

which allows to evaluate the LDOS at a given site (or any linear combination of site orbitals). Since $\mathcal{H}$ is purely offdiagonal and $\mathcal{T}_{3}$ is bipartite (and therefore only contains even loops), the diagonal $a_{n}$ terms vanish (i.e. the odd moments). Notice that in this case, the orbital $|n\rangle$ encompasses all the sites reached from $|0\rangle$ at least after $n$ jumps, and can be interpreted in terms of shell orbitals. If one of the $b_{n}$ vanishes, the effective chain becomes finite and the LDOS is discrete. It also implies that a wavepacket initially localized in the tiling at the origin site will only spread over a finite set of sites belonging to the mutually coupled shells.

We first choose the initial orbital $|0\rangle$ at a 3 -fold coordinated site and apply the recursion procedure. One then easily obtains: $b_{1}^{3}=\sqrt{3}, b_{2}^{3}=D_{2} / \sqrt{3}$ and $b_{3}^{3}=D_{3} / D_{2}$ where:

$$
\begin{aligned}
& D_{2}=\left(12 \cos ^{2}(\pi f)+9\right)^{1 / 2} \\
& D_{3}=\left(24 \cos ^{2}(\pi f)+12(\cos (\pi f)+\cos (3 \pi f))^{2}\right)^{1 / 2} .
\end{aligned}
$$

It is clear that $b_{3}^{3}=0$ for $f=1 / 2$, and that the LDOS reduces to the following three eigenvalues $0, \pm \sqrt{6}$. A similar derivation for the 6 -fold coordined site leads to: $b_{1}^{6}=\sqrt{6}, b_{2}^{6}=2 \cos (\pi f)$. For $f=1 / 2, b_{2}^{6}$ also vanishes and the LDOS reduces to $\pm \sqrt{6}$ (see figure 3 ).

We now proceed to describe the quantum dynamics on $\mathcal{T}_{3}$. Owing to the superposition principle, we only consider initial distributions strictly localized on each type site, given that any wavepacket behaviour can be described in terms of these individual evolutions. For a generic value of $f$, the initially localized electron wavefunction spreads with time and asymptotically vanishes at the origin, the time dependance of this phenomenon being determined by the nature of the eigenspectrum. For $f=1 / 2$, the quantum dynamics can be derived in 
closed forms and it displays a very different behaviour since the system remains localized inside a restricted region. Indeed, an initial distribution localized on a $A$ site is completely "trapped" inside its surrounding star $\left(b_{2}^{6}=0\right)$ so that, at any time, there will only be nonvanishing components of the wavefunction at the origin and on the first shell. This oscillating motion also occurs for a wavepacket initially localized on a $B($ or $C)$ site $\left(b_{3}^{3}=0\right)$, but is here confined inside a larger neighborhood (see figure 3). Consequently, for $f=1 / 2$, any wavepacket on $\mathcal{T}_{3}$ has a limited extent.

This extreme localization in a magnetic field can be simply understood in terms of Aharonov-Bohm interferences around the rhombi; we therefore call the above confined regions $\mathcal{A}$. $\mathcal{B}$. cages. More precisely, for a given tiling embedded in a magnetic field $\mathbf{H}$, we associate to any site $|i\rangle$, an $\mathcal{A}$. $\mathcal{B}$. cage $\mathcal{C}_{i}^{H}$ defined as the set of sites reached by a wavepacket initially localized on $|i\rangle$. Note that $\mathcal{C}_{i}^{H}$ is generically infinite, but specific values of the magnetic field $\left(f=1 / 2\right.$ for $\left.\mathcal{T}_{3}\right)$ can leave it bounded. In this case, the cage can also be characterized by its boundary sites, where completely destructive interferences disconnect it from the rest of the tiling. For a given structure, it is sufficient to characterize the inequivalent site cages, and several cases can occur:

- All cages are unbounded for any $f$. Ex: Square lattice, triangular lattice, honeycomb.

- Some cages are bounded for particular values of $f$. Ex: Star patterns in Penrose tilings and octagonal tilings (see below).

- For the same values of $f$, all cages are simultaneously bounded.

The simplest example of the latter case is encountered on $\mathcal{T}_{3}$ for $f=1 / 2$, but it can also occur for other tilings. Let us pay attention to $\mathcal{T}_{4}$ represented figure in 4 which is an approximant with 14 sites per unit cell of the octagonal quasiperiodic tiling. This tiling contains three types of faces (large square, small square, and parallelogram), but only two different areas.

The previous recursion analysis can be done on the five different types of site (A, B, C, D, E) and one can prove that for $f=1 / 2$, all the site cages are bounded (in this case $f$ refers to the magnetic flux per small area). Thus, the eigenspectrum is also discrete and the quantum dynamics is similar to that discussed on $\mathcal{T}_{3}$ (bounded extension of any wavepacket, pulsating states, ...). It is also possible to continuously deform $\mathcal{T}_{4}$, keeping the same connectivity, until all the edges have the same length, and the star around $A$ becomes 8 -fold symmetric. But any metrical transformation changes the nature of the spectrum and the dynamics, so that only some site cages remains bounded $\left(\mathcal{C}_{A}^{H}\right.$ for $\left.f=1 / 2\right)$. In addition, when all the edge lengths are equal, the areas are incommensurate and the energy spectrum is no longer periodic with $f$.
This loss of periodicity does always occur when two tile areas are incommensurate as it is the case in quasiperiodic tilings. Indeed, consider the closed paths in the tiling contributing, for instance, to the fourth moment of the LDOS at a generic site. Among them, only the non self-retracing ones are field dependent, and lead to cosine terms whose arguments depend on the circumnavigated rhombi areas. In those tilings, the spectrum becomes then quasiperiodic with $f$ as remarked in [10] [17].

Concerning quasiperiodic tilings, some local configurations display interesting characteristics in the presence of a magnetic field. As for $\mathcal{T}_{3}$ and $\mathcal{T}_{4}$, star-like patterns can be encountered, providing canonical example of $\mathcal{A}$. $\mathcal{B}$. cages. An easy way to understand the properties of such an environment is to use the symmetry group of the cluster. For convenience, we only consider p-fold symmetric stars $(2 p+1$ sites) (see figure $3(\mathrm{p}=6))$, whose first shell sites are 3 -fold coordinated and where all the edges have the same length. In the absence of a magnetic flux, those stars remains unchanged under $C_{p v}$ transformations, but the magnetic field breaks the reflection invariance. Finally, one can only deal with the $C_{p}$ rotation group. We denote the $p$ one-dimensional irreductible representations by $\Gamma_{i}(i=0, p-1)$. If we consider the symmetrical representation $\Gamma_{0}$, one can show that the LDOS at the star center reduces to $\varepsilon^{ \pm}= \pm \sqrt{p}$, as soon as $f=1 / 2$. This result is nothing but a generalization of the above recursion study on the $\mathcal{T}_{3} A$ site, to any $p$-order symmetry. Consequently, the dynamical properties are also comparable and one faces a simple example of $\mathcal{A}$. $\mathcal{B}$. cage that appears in quasiperiodic rhombus tilings (Penrose $(p=5,10)$, octagonal $(p=8))$. A close investigation of the other $p-1$ representations shows that when $f=m / p(m=1, p-1)$, one can identify confined eigenstates associated to $\varepsilon=0$, similar to those existing for $f=0$. Along the same line, one can prove that there exist strictly localized states in the clusters studied by Kohmoto et al. [18], for any values of the magnetic field. Some of these states persist in the infinite tiling and have actually been numerically observed by Schwabe et al. [10].

It is also important to clarify the effect of disorder on this field induced localization. Queerly, one expects the randomness to alter and even destroy the phase matching essential for this localization effect. A standard way to disorder a system is to randomly modulate the hopping terms without changing the tiling configuration; but a different approach consist in flipping the rhombic tiles as in the Random Tiling Model 19. In $\mathcal{T}_{3}$, a finite number of such flips should neither affect the discrete nature of the spectrum nor the confined diffusion process. However, a finite density of flips may change those properties.

Finally, a natural question arises about the possibility to experimentally observe this very strong localization phenomenon. We should first remark that $\mathcal{A}$. $\mathcal{B}$. cages are sensitive to various kinds of disorder which are likely 
to occur in real systems: fluctuations in the tile areas and in the transmission matrix along the edges are strongly relevant, but unlikely to fully destroy the localized nature of the eigenstates. We may just conjecture a strange non-monotonous variation of the localization length as a function of disorder strength.

A certainly interesting experiment would be to investigate the magnetic vortex lattice in a superconducting wire network with the $\mathcal{T}_{3}$ geometry. Indeed, the unusual nature of the linearized Ginzburg-Landau equation eigenstates is to be reflected on the behavior of the solutions in the presence of the non-linear term. A crucial issue would be to determine whether there is a unique optimal lattice structure, or a glassy type of vortex-solid, or even a disordered vortex-liquid. These various possibilities are still debated in simpler geometries [20]. We believe that the systems presented here are good candidates for multiple ground state configurations, but the nature of the energy barriers connecting them is certainly a challenging open problem.

\section{ACKNOWLEDGMENTS}

We would like to thank Cl. Aslangul, B. Delamotte, D. Mouhanna and B. Pannetier for fruitful discussions.

[1] H. L. Stormer et al., Phys. Rev. Lett. 50, 1953 (1983).

[2] H. W. Jiang et al., Phys. Rev. Lett. 65, 663 (1990); V. G. Goldman et al., Phys. Rev. Lett. 65, 2189 (1990).

[3] K. von Klitzing, G. Dorda and M.Pepper, Phys. Rev. Lett. 45, 494 (1980).

[4] A. Mac Kinnon, L. Schweitzer and B. Kramer, Surf. Science 142, 189 (1984).

[5] M. Y. Azbel, JETP 19, 634 (1964).

[6] D. R. Hofstadter, Phys. Rev. B 14, 2239 (1976).

[7] B. Pannetier, J. Chaussy and R. Rammal, J. Phys. Lett. 44, 853 (1983).

[8] F. H. Claro and G. H. Wannier, Phys. Rev. B 19, 6068 (1979).

[9] R. Rammal, J. Phys. 46, 1345 (1985).

[10] A. Behrooz et al., Phys. Rev. Lett. 57, 368 (1986); K. Springer and D. van Harlingen, Phys. Rev. B 36, 7273 (1987); H. Schwabe, G. Kasner and H. Böttger, Phys. Rev. B 56, 8026 (1997).

[11] B. Douçot, et al., Phys. Rev. Lett. 57 1235, (1986); J. M. Gordon et al., Phys. Rev. Lett. 56 2280, (1986).

[12] F. Nori and Q. Niu, Phys. Rev. B 39, 2134 (1989).

[13] Y. Aharonov and D. Bohm, Phys. Rev. 115, 485 (1959).

[14] B. Sutherland, Phys. Rev. B 34, 5208 (1986).

[15] R.E. Peierls, Z. Phys. 80, 763 (1933).

[16] R. Haydock, V. Heine and M. J. Kelly, J. Phys. C. 8, 2591 (1975).
[17] U. Grimm, F. Gagel and M. Schreiber, Proc. of the $6^{\text {th }}$ Int. Conf. on Quasicrystals (Ed. S. Takeuchi and T. Fujiwara, World Scientific) p.188, (1998).

[18] M. Kohmoto and B. Sutherland, Phys. Rev. Lett. 56 2740, (1986).

[19] V. Elser, Phys. Rev. Lett. 54 1730, (1985).

[20] C. Denniston and C. Tang, Phys. Rev. Lett. 79 451, (1997); P. Gupta, S. Teitel and M. P. Gingras, Phys. Rev. Lett. 80 105, (1998).

\section{Figure Captions}

1. A piece of $\mathcal{T}_{3}$ lattice embedded in a perpendicular magnetic field $\mathbf{H} ; a$ is the rhombus edge length.

2. $\mathcal{T}_{3}$ butterfly-like energy spectrum versus $f$.

3. Effective semi-infinite chain (recursion framework), associated to $\mathcal{T}_{3}$ local environments (6-fold site (left), 3-fold site (right)).

4. A piece of the $\mathcal{T}_{4}$ periodic tiling. The five different types of sites are depicted

(A, B, C, D, E). 


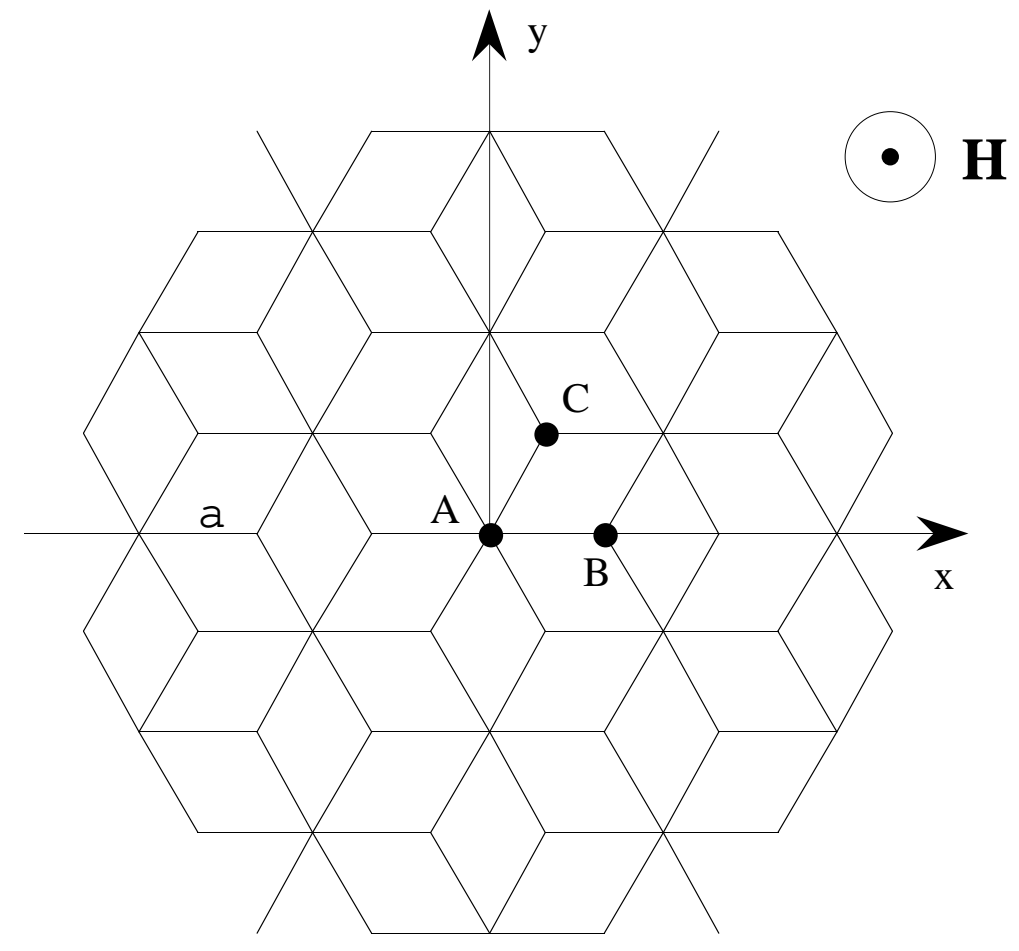




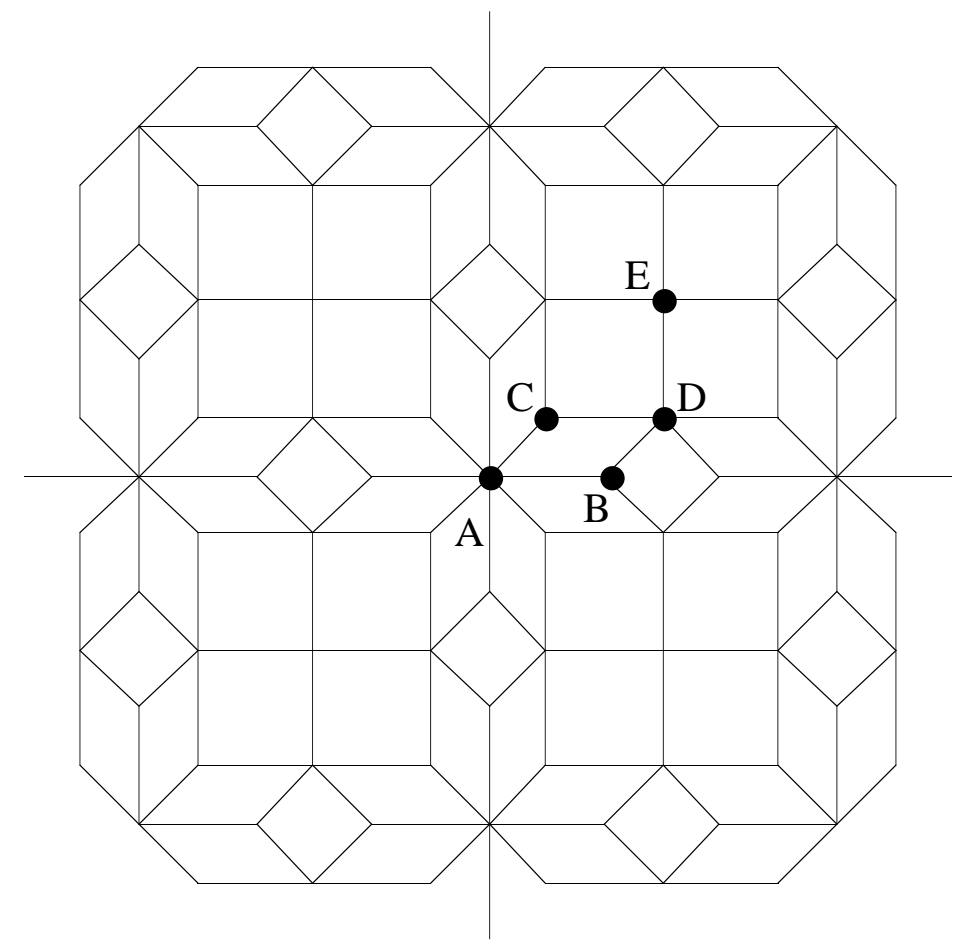




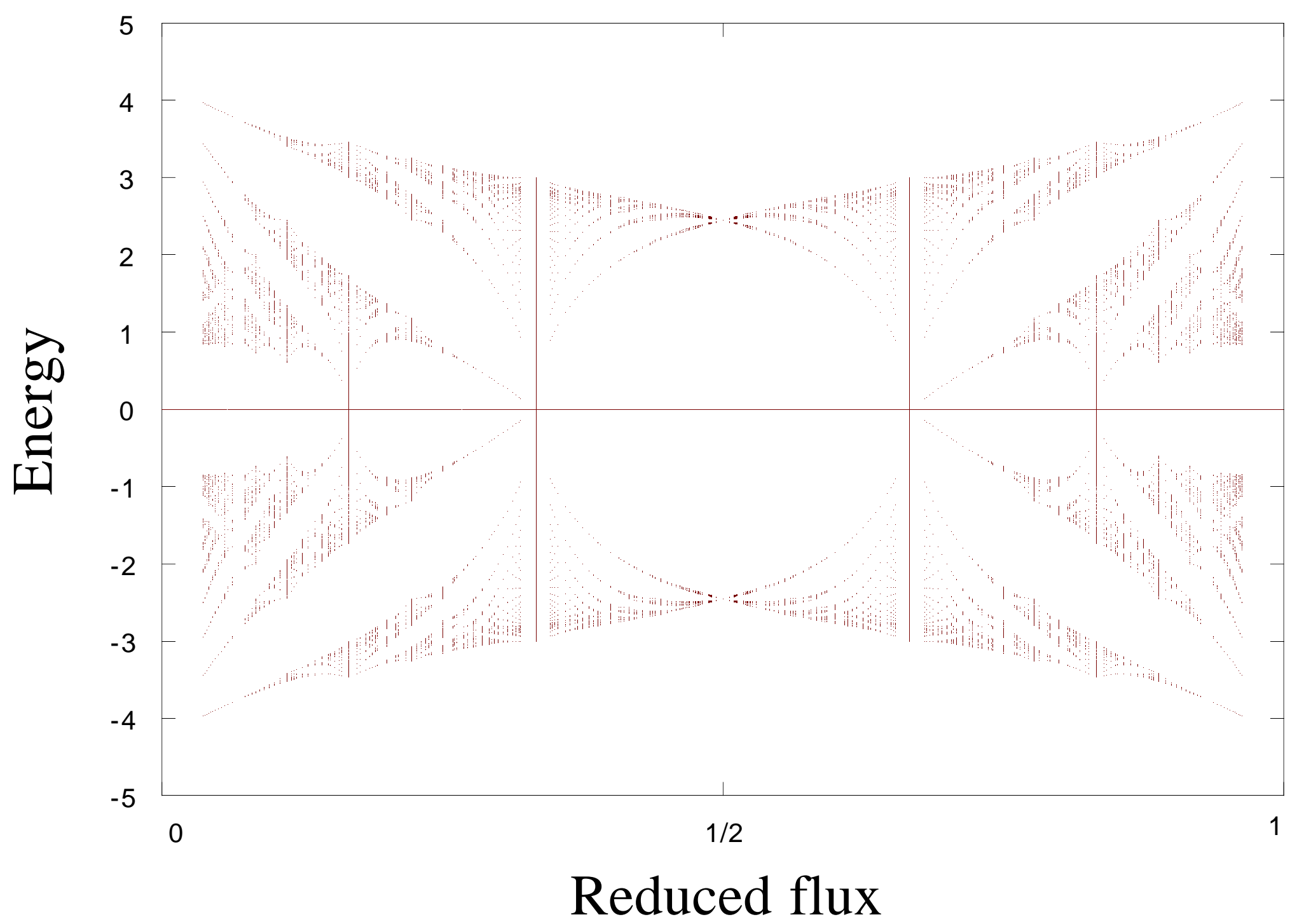


